\title{
Short training videos for hands-only CPR improve bystander responses in out-of-hospital cardiac arrest scenarios
}

Bystander cardiopulmonary resuscitation $(\mathrm{CPR})$ is known to improve the survival chances of individuals who experience out-of-hospital cardiac arrest (OHCA); however, many OHCA witnesses do not attempt CPR. A new study demonstrates that use of a short training video for hands-only CPR (chest compressions with no mouth-to-mouth ventilation) can result in improved bystander CPR rates.

Even $60 \mathrm{~s}$ videos were associated with beneficial effects.

"We are deeply concerned with the low rates of bystander CPR and are committed to measuring and improving [these] rates and survival from OHCA," explains Bentley Bobrow, one of the investigators. "We had seen significant increases in compression-only CPR when we used brief training videos and wanted to formally study these."

The investigators therefore created an OHCA scenario, using a manikin, and compared the responses of 51 untrained individuals with those of individuals who had viewed videos $60 \mathrm{~s}(n=95), 5 \mathrm{~min}$ $(n=99)$, or $8 \min (n=91)$ in length. "The AHA has studied their 22-minute CPR Anytime videos," explains Dr Bobrow, "but we were interested in even more brief video training, which could be used to reach the public in multiple different venues and formats." Those who viewed the $8 \mathrm{~min}$ video before testing also practiced $\mathrm{CPR}$ skills on a manikin during the viewing. Half of the trained study participants were tested within $3 \mathrm{~h}$ of viewing the videos and half were tested 2 months later.

Overall, 12 (24\%) of the control group and $2(4 \%)$ of the study participants who were tested 2 months after watching the $60 \mathrm{~s}$ video did not attempt CPR when exposed to the OHCA scenario. All other study participants did attempt resuscitation. The median compression rate and compression depth for the control group were substantially lower than those for all other experimental groups (62 compressions/min versus 90-99.5 compressions/min, and $30 \mathrm{~mm}$ versus $41-48 \mathrm{~mm}$, respectively). Delay in testing had no significant effect on these measures in the trained individuals.

The investigators believe that this is the first randomized, controlled study evaluating the efficacy of ultra-brief handsonly CPR training for the general public. On the basis of the findings, Dr Bobrow concludes that "ultra-brief hands-only CPR videos hold great promise to increase bystander CPR rates and save lives."

Bryony M. Mearns

Original article Bobrow, B. J. et al. The effectiveness of ultrabrief and brief educational videos for training lay responders in hands-only cardiopulmonary resuscitation: implications for the future of citizen cardiopulmonary resuscitation training. Circ. Cardiovasc. Qual. Outcomes 4, 220-226 (2011) 